\title{
Mitarbeiterinnen und Mitarbeiter
}

Birn, Ruth Bettina, Dr., 59 Wilton Crescent, Ottawa, Ont K1S 2T6, Canada $\quad(228,229)$

Brandl, Ulrich, Dr., Archäologe, Bürogemeinschaft asm, Dinslakener Landstr. 9, D-46483 Wesel (ubrandl@web.de) (261)

Chiari, Bernhard, Dr., Militärgeschichtliches Forschungsamt (MGFA), Potsdam (277)

Deisenroth, Karlheinz, Brombergstr. 24, 79102 Freiburg i.Br. $\quad(269,272)$

Dülffer, Jost, Prof. Dr., Universität zu Köln, Historisches Seminar, Albertus-Magnus-Platz, 50923 Köln (212)

Echternkamp, Jörg, Dr. phil., MGFA, Potsdam (JoergEchternkamp@bundeswehr.org) (292)

Etschmann, Wolfgang, Dr., Militärgeschichtliche Forschungsabteilung / Heeresgeschichtliches Museum, Wien (155)

Föllmer, Moritz, Dr. des., Institut für Geschichtswissenschaften, Humboldt-Universität zu Berlin (1)

Fuchs, Konrad, Prof. Dr., Johannes Gutenberg-Universität Mainz, Historisches Seminar IV, 55122 Mainz (260)

Geßner, Klaus, Dr., Abteilungsleiter am Brandenburgischen Landeshauptarchiv (Potsdam) (175)

Groß, Gerhard P., Dr. MGFA, Potsdam (286)

Hartmann, Karl, Dr. phil. (91)

Hass, Gerhart, Prof. Dr., Rangsdorf bei Berlin (235)

Heitmann, Clemens, M.A., MGFA, Potsdam (240)

Heuchling, Jürgen O.K.H., M.A., Dipl.-Kfm., Oberstleutnant d.R. (295)

Hillmann, Jörg, Dr., FKpt., Universität der Bundeswehr Hamburg, Fachbereich Pädagogik, Seminar für Geschichtswissenschaft $(217,257,264)$

Hüllbüsch, Ursula, Dr. (245)

Kehrig, Manfred, Dr., Ltd. Archivdirektor, Oberst a.D. und d.R., Leiter Bundesarchiv-Militärarchiv (268)

Kehrt, Christian, Tucholskystr. 26, 10117 Berlin (ckehrt@hotmail.com) (49)

Kellmann, Axel, M.A., Doktorand, Klettenberggürtel 79, 50939 Köln (223)

Kröger, Martin, Dr., Rathausstr. 24, 51143 Köln (211)

Möllers, Heiner, Dr., Major, Luftwaffenamt, Köln (289)

Moldenhauer, Harald, M.A., Bonn, Doktorand bei Prof. Hans Hecker an der Heinrich-Heine-Universität Düsseldorf (232)

Moll, Martin, Dr., Wilhelm-Kienzlgasse 33, A-8010 Graz (243)

Morlang, Thomas, Mielesheide 6, 45133. Essen (287)

Müller, Klaus-Jürgen, Dr. phil., Hanfstieg 25, 22549 Hamburg $\quad(279,304)$

Neitzel, Sönke, Dr. habil., HD, Johannes Gutenberg-Universität Mainz, Historisches Seminar IV, Saarstr. 21, 55099 Mainz (225)

Neumann, Alexander, Doktorand, Erlenweg 6, 79115 Freiburg (237)

Overmans, Rüdiger, Dr. rer. pol. et phil., MGFA, Potsdam/Universität Freiburg, Brunnmatten 8, 79108 Freiburg (274, 275, 301, 302)

Pavlenko, Irina, Kandidatin der historischen Wissenschaften, Abteilungsleiterin im Zentralen Staatsarchiv für gesellschaftliche Vereinigungen der Ukraine (Kiew) (73)

Pelizaeus, Ludolf, Dr., Johannes Gutenberg-Universität, Mainz (262)

Pröve, Ralf, Dr. phil. habil., Privatdozent für Neuere und Neueste Geschichte, Lloyd-G.-Wells-Str. 29, 14163 Berlin (267)

Rahn, Werner, Dr., Kapitän zur See a.D., Lüdickeweg 9c, 14089 Berlin (141) 
Raß, Christoph, Dr. rer. pol., Wiss. Ass. am Lehr- und Forschungsgebiet Wirtschaftsund Sozialgeschichte der RWTH Aachen, Templergraben 83, 52062 Aachen (206)

Rogg, Matthias, Dr., Major, MGFA, Potsdam (195)

Schmidt, Gustav, Prof. Dr., Lehrstuhl Internationale Politik, Fakultät für Sozialwissenschaft, und hospitiertes Mitglied der Fakultät für Geschichtswissenschaft, Ruhr-Universität Bochum (249)

Schmidt, Jürgen, M.A., Doktorand, Albert-Buchmann-Str. 2, 16515 Oranienburg $(282,283,284)$

Scholz, Michael F., Ph.D., Associate Professor History, Gotland University, 62167 Visby, Schweden $(291,297)$

Sieg, Dirk, M.A., Bonn $(294,295)$

Stegmann, Natali, Dr., Osteuropa-Historikerin (Bremer.Stegmann@t-online.de) (25)

Steinert, Marlis, Prof. em. Dr. (233)

Stübig, Heinz, Prof. Dr., Institut für Erziehungswissenschaft, Philipps-Universität Marburg, 35032 Marburg (265)

Vogel, Thomas, Dr. phil., Major, MGFA, Potsdam (220) 